\section{Überaktive Blase: Solifenacin und Kognition}

— Viele Ärzte vermeiden es, Anticholinergika bei älteren Menschen mit überaktiver Blase (overactive bladder, OAB) einzusetzen. Größter Vorbehalt: Die Wirkstoffe könnten die Kognition beeinträchtigen. Eine aktuelle Studie von Adrian Wagg, Edmonton, Canada, liefert konkrete Daten: Wagg und seine Kollegen konnten in der SENIOR-Studie bei 23 Probanden (medianes Alter: 79 Jahre) mit milder eingeschränkter Kognition zeigen, dass keine statistisch signifikanten Unterschiede bei den fünf getesteten Kognitionsparametern zwischen dem Anticholinergikum Solifena- cin $5 \mathrm{mg}\left(\right.$ Vesikur $\left.^{\oplus}\right)$ und Placebo auftraten [Wagg a et al. Eur Urol. 2013; 64: 74-81]. „Diese Ergebnisse sollten uns Ärzten mehr Sicherheit bei der Behandlung der OAB bei älteren Patienten geben", sagte Prof. Ruth Kirschner-Hermanns, Leiterin der Neurourologie der Universitätsklinik Bonn im Gespräch mit der Ärzte Zeitung.

Spätestens seit der EPIC-Studie aus dem Jahre 2005 weiß man, dass die OAB eine sehr häufige Blasenfunktionsstörung ist, die mit dem Alter deutlich zunimmt. Typische Symptome gemäß International Continence Society (ICS) sind überfallarti-

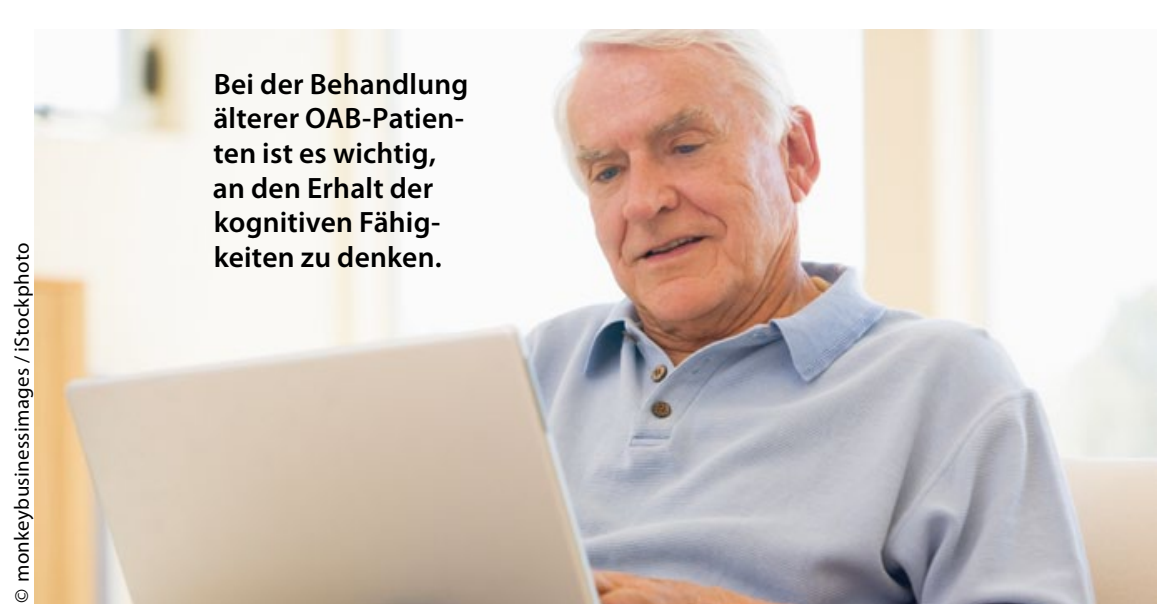

ger Harndrang, Pollakisurie und Nykturie. Gerade der Harndrang kann bei Älteren zur erhöhten Komorbidität führen, indem er beispielsweise Stürze begünstigt.

Neben nicht medikamentösen Therapiemöglichkeiten wie Blasen- und Toilettentraining gelten Anticholinergika gemäß der Leitlinien nationaler und internationaler Fachgesellschaften als Therapeutikum der Wahl mit hohem Evidenz-Level bei der OAB. "Mit dem richtigen Augenmaß auf die besondere Situation Älterer muss der mögliche Nutzen einer anticholinergen Therapie abgeschätzt werden", so Kirschner-Hermanns. Die Wirkstoffe seien gemäß Leitlinie der Deutschen Gesellschaft für Geriatrie (DGG) zur symptomatischen Therapie der Dranginkontinenz geeignet: „Unter Beachtung der Kontraindikationen und Nebenwirkungen stellen Anticholinergika eine wirksame Therapie dar", so die Expertin. Sie reduzieren die Detrusorkontraktilität, verringern den Detrusordruck und führen zu einer gesteigerten Blasenkapazität. Dadurch bessert sich die Drangsymptomatik, so dass Inkontinenzepisoden und die damit verbundenen Folgeereignisse seltener auftreten.

Constanze Löffler

Nach Informationen von

Astellas, München

\title{
Erektile Dysfunktion: auch an Testosteronmangel denken!
}

_ Erektionsprobleme wie die erektile Dysfunktion (ED) gelten als Vorbote kardiovaskulärer Störungen, ein Testosterondefizit ist eng assoziiert mit Begleiterkrankungen. „ED-Patienten sind oft Hypertoniker oder Männer mit Diabetes mellitus oder beidem", erklärte Dr. Horst Hohmuth, Ulm, beim Symposium „Mann Vital“ im April in Hannover.

Mit dem Anstieg der Lebenserwartung wird künftig auch der Altershypogonadismus an Bedeutung gewinnen: Er ist assoziiert mit verminderter Lebensqualität, erhöhter Morbidität (Adipositas, metabolischem Syndrom, Diabetes mellitus, kardiovaskulären Erkrankungen, Stürze, Osteoporose) und Mortalität. Bei gesichertem
Testosterondefizit - körperliche Symptome plus labortechnischer Nachweis - verbessert eine Testosterontherapie die Sexualfunktion, die Stimmungslage steigt, die fettfreie Muskelmasse nimmt zu, die Muskelkraft in Beinen und Armen wächst, langfristig steigt auch die Knochendichte. Der Einsatz eines Testosteron-Gels (etwa Androtop ${ }^{\circledR} \mathrm{Gel}$ ) hat dabei eindeutige Vorteile gegenüber anderen Applikationsformen, wie Prof. Armin Heufelder, München, darlegte: Es ist individuell dosierbar, führt bei schnellem Wirkungseintritt zu stabilen Testosteronwerten im Normbereich. Hautirritationen sind selten. Und sollte dies notwendig sein, kann das Gel rasch abgesetzt werden.
Bei einem Teil der Non-Responder auf PDE5-Hemmer liegt ein Testosteronmangel vor. Sie sprechen bei Kombination mit Testosteron an, wenn ein echtes Defizit besteht $(\leq 3$ $\mathrm{ng} / \mathrm{ml}$ ). Je niedriger der Basiswert, desto überzeugender war der Effekt in einer Studie mit $1 \%$ igem Testosteron-Gel.

Die Kombination mit Testosteron-Gel (Androtop ${ }^{\oplus} \mathrm{Gel}$ ) kann auch bei ED-Patienten mit niedrig-normalem Testosteronspiegel $(\leq 4$ $\mathrm{ng} / \mathrm{ml}, 14 \mathrm{nmol} / \mathrm{l}$ ) zum Erfolg führen, die auf eine dreimonatige Sildenafil-Medikation nicht angesprochen hatten [Rosenthal BD et al. Urology 2006; 67: 571-4]. Nach vier Wochen normalisierten sich die Hormonspiegel, nach 16 Wochen war die Potenz deutlich verbessert.

Nach Informationen von Dr. Kade/Besins, Berlin 